\title{
On Removable Sets of Solutions of Neuman Problem for Quasilinear Elliptic Equations of Divergent Form
}

\author{
Tahir S. Gadjiev, Orxan S. Aliyev \\ Institute of Mathematics of Academy Sciences, Baku, Azerbaijan \\ Email: tgadjiev@mail.az
}

Received November 21, 2012; revised December 25, 2012; accepted January 3, 2013

\begin{abstract}
In this paper we consider a nondivergent elliptic equation of second order whose leading coefficients are from some weight space. The sufficient condition of removability of a compact with respect to this equation in the weight space of Hölder functions was found.
\end{abstract}

Keywords: Removable; Elliptic; Degenerate; Neumann Problem

\section{Introduction}

Let $D$ be a bounded domain situated in $n$-dimensional Euclidean space $E_{n}$ of the points $x=\left(x_{1}, \cdots, x_{n}\right)$, $n \geq 3, \partial D$ be its boundary. Consider in $D$ the following elliptic equation

$$
\left\{\begin{array}{l}
L u=\sum_{i, j=1}^{n} \frac{\partial}{\partial x_{i}}\left(a_{i j}(x) \frac{\partial u}{\partial x_{j}}\right)+\sum_{i=1}^{n} b_{i}(x) u_{x_{i}} \\
\quad+c(x) u+b(x, u, \nabla u)=0 \\
\left.u\right|_{\partial D / E}=0
\end{array}\right.
$$

in supposition that $\left\|a_{i j}(x)\right\|$ is a real symmetric matrix, moreover

$$
\begin{aligned}
& \gamma|\xi|^{2} \omega(x) \leq \sum_{i, j=1}^{n} a_{i j}(x) \xi_{i} \xi_{j} \leq \gamma^{-1} \omega(x)|\xi|^{2} ; \\
& \xi \in E_{n}, x \in D, \\
& a_{i j}(x) \in C_{\omega}^{1}(\bar{D}) ; i, j, 1, \cdots, n, \\
& \left|b_{i}(x)\right| \leq b_{0} ;-b_{0} \leq c(x) \leq 0 ; i=1, \cdots, n ; x \in D . \\
& |b(x, u, \nabla u)| \leq g(u) \omega(x)|\nabla u|, \\
& \int_{0}^{a} g(u) \mathrm{d} u<\infty, a<\infty .
\end{aligned}
$$

Here $g(x)$ is non-negative function from

$$
u, u_{i}=\frac{\partial u}{\partial x_{i}}, u_{i j}=\frac{\partial^{2} u}{\partial x_{i} \partial x_{j}} ; i, j=1, \cdots, n ; \gamma \in(0,1]
$$

and $b_{0} \geq 0$ are constants. Besides we'll assume that the minor coefficients of the operator $L$ are measurable in $D$. Let $\lambda \in(0,1)$ be some number.

The compact $E \subset \bar{D}$ is called removable with respect to the Equation (1) in the space $C_{\omega}^{\lambda}(D)$ if from

$$
L u=0, x \in D / E ;\left.u\right|_{\partial D / E}=0 ; u(x) \in C_{\omega}^{\lambda}(D)
$$

it follows that $u(x) \equiv 0$ in $D$.

\section{Auxiliary Results}

The paper is organized as follows. In Section 2, we present some definitions and auxiliary results. In Section 3 we give the main results of the sufficient condition of removability of compact.

The aim of the given paper is finding sufficient condition of removability of a compact with respect to the Equation (1) in the space $C_{\omega}^{\lambda}(D)$. This problem have been investigated by many researchers. For the Laplace equation the corresponding result was found by L. Carleson [1]. Concerning the second order elliptic equations of divergent structure, we show in this direction the papers [2,3]. For a class of non-divergent elliptic equations of the second order with discontinuous coefficients the removability condition for a compact in the space $C^{\lambda}(D)$ was found in [4]. Mention also papers [5-9] in which the conditions of removability for a compact in the space of continuous functions have been obtained.The removable sets of solutions of the second order elliptic and parabolic equations in nondivergent form were considered in [10-12]. In [13], T. Kilpelainen and X. Zhong have studied the divergent quasilinear equation without minor members, proved the removability of a compact. Removable sets for pointwise solutions of elliptic partial differential equations were found by $\mathrm{J}$. 
Diederich [14]. Removable singularities of solutions of linear partial differential equations were considered in $\mathrm{R}$. Harvey, J. Polking paper [15]. Removable sets at the boundary for subharmonic functions have been investigated by B. Dahlberg [16]. Also we mentioned the papers of A.V.Pokrovskii [17,18].

In previous work, authors considered Direchlet problems for linear equations in some space of functions. In this work we consider Newman problem for quasilinear equations and sufficient conditions of removability of a compact in the weight space of Holder functions is obtained. The application value of the research in many physic problems.

Denote by $B_{R}(z)$ and $S_{R}(z)$ the ball $\{x:|x-z|<R\}$ and the sphere $\{x:|x-z|=R\}$ of radius $R$ with the center at the point $z \in E_{n}$ respectively. We'll need the following generalization of mean value theorem belonging to E.M. Landis and M.L. Gerver [8] in weight case.

Lemma. Let the domain $D$ be situated between the spheres $S_{R}(0)$ and $S_{2 R}(0)$, moreover the intersection $\partial D \cap\{x: R<|x|<2 R\}$ be a smooth surface. Further, let in $\bar{D}$ the uniformly positive definite matrix

$$
\left\|a_{i j}(x)\right\| ; i, j=1, \cdots, n
$$

and the function $u(x) \in C^{2}(D) \cap C_{\omega}^{1}(\bar{D})$ be given. Then there exists the piece-wise smooth surface $\Sigma$ dividing in $G$ the spheres $S_{R}(0)$ and $S_{2 R}(0)$ such that

$$
\int_{\Sigma} \omega\left|\frac{\partial u}{\partial v}\right| \mathrm{d} s \leq \underset{D}{\operatorname{Oosc} u} \cdot \frac{\omega(D)}{R^{2}} .
$$

Here $K>0$ is a constant depending only on the matrix $\left\|a_{i j}(x)\right\|$ and $n, \frac{\partial u}{\partial v}$ is a derivative by a conormal determined by the equality

$$
\frac{\partial u(x)}{\partial v}=\sum_{i, j=1}^{n} a_{i j}(x) \frac{\partial u(x)}{\partial x_{i}} \cos \left(\bar{n}, x_{j}\right)^{\frac{1}{2}},
$$

where $\cos \left(\bar{n}, x_{j}\right) ; j=1, \cdots, n$ are direction cosines of a unit external normal vector to $\Sigma$.

Proof. Let $G \subset R^{n}$ be a bounded domain $f(x) \in C^{2}(G)$. Then for any there exists a finite number of balls $\left\{B_{r_{v}}^{x_{v}}\right\}, v=1,2, \cdots, N$ which cover $Q_{f}$ and such that if we denote by $S_{v}$, the surface of $v$-th ball, then

$$
\sum_{v=1}^{N} \int_{S_{v}} \omega(x)|\nabla f| \mathrm{d} x<\varepsilon .
$$

Decompose $O_{f}$ into two parts: $O_{f}=O_{f}^{\prime} \cup O_{f}^{\prime \prime}$, where $O_{f}^{\prime}$ is a set of points $O_{f}$ for which $\nabla^{2} f \neq 0, O_{f}^{\prime \prime}$ is a set of points for which $\nabla^{2} f=0$.

The set $O_{f}^{\prime}$ has $n$-dimensional Lebesque measure equal zero, as on the known implicit function theorem, the $O_{f}^{\prime}$ lies on a denumerable number of surfaces of dimension $n-1$. If we use the absolute continuity of integral

$$
\omega(D)=\int_{D} \omega(x) \mathrm{d} x
$$

with respect to Lebesque measure $D$ and above said we get that the set $O_{f}^{\prime}$ may be included into the set $D$ for which $\omega(D)<\eta, \eta>0$ will be choosen later. Let for each point $x \in O^{\prime} f$ there exist such $r_{x}$ that $B_{r_{x}}^{x}$ and $B_{6 r_{x}}^{x}$ are contained in $D \subset G$. Then

$$
\int_{5 r_{x}}^{6 r_{x}} \mathrm{~d} r \int_{S_{r}^{x}} \omega(\sigma) \mathrm{d} \sigma \leq \omega\left(B_{6 r_{x}}^{x}\right)
$$

therefore there exists such $5 r_{x} \leq t \leq 6 r_{x}$ that

$$
r_{x} \int_{S_{r}^{x}} \omega(\sigma) \mathrm{d} \sigma \leq \omega\left(B_{6 r_{x}}^{x}\right) .
$$

Then

$$
\begin{aligned}
& \int_{S_{r}^{x}} \omega(\sigma)|\nabla f| \mathrm{d} \sigma \leq C t \int_{S_{r}^{x}} \omega(\sigma) \mathrm{d} \sigma \\
& \leq(6 C) r\left(r_{x} \int_{S_{r}^{x}} \omega(\sigma) \mathrm{d} \sigma\right) \\
& \leq(6 C) \omega\left(B_{6 r_{x}}^{x}\right) \leq(6 C) \gamma^{-3} \omega\left(B_{r_{x}}^{x}\right) \leq c_{0} \omega\left(B_{t / 5}^{x}\right),
\end{aligned}
$$

where

$$
C=\sup _{D}\left|\nabla^{2} f\right|, \alpha=\operatorname{diam} G, c_{0}=(6 C) \gamma^{-3} .
$$

Now by a Banach process ([4], p.126) from the ball system $\left\{B_{t / 5}^{x}\right\}$ we choose such a denumerable number of not-intersecting balls $\left\{B_{t_{v} / 5}^{x_{v}}\right\}, v=1,2, \cdots, N$ that the ball of five times greater radius $\left\{B_{t_{v}}^{x_{v}}\right\}$ cover the whole $O_{f}^{\prime} \quad$ set. We again denote these balls by

$$
\left\{B_{t_{v} / 5}^{x_{v}}\right\}, v=1,2, \cdots, N
$$

and their surface by $S_{v}^{\prime}$. Then by virtue of (4)

$$
\sum_{v=1}^{\infty} \int_{S_{v}^{\prime}} \omega(\sigma)|\nabla f| \mathrm{d} \sigma \leq C_{0} \omega(G)<C_{0} \eta .
$$

Now let $x \in O_{f}^{\prime \prime}$. Then

$$
\int_{5 r_{x}}^{6 r_{x}} \mathrm{~d} r \int_{S_{r}^{x}} \omega(\sigma) \mathrm{d} \sigma \leq \omega\left(B_{6 r_{x}}^{x}\right)
$$

Therefore there exists such $5 r_{x} \leq t \leq 6 r_{x}$ that

$$
r_{x} \int_{S_{r}^{x}} \omega(\sigma) \mathrm{d} \sigma \leq \omega\left(B_{6 r_{x}}^{x}\right) .
$$

Assign arbitrary $\eta>0$. By virtue of that $|\nabla f|_{S_{t}^{x}} \leq \eta \cdot t$, 
for sufficiently small $t$ we have

$$
\begin{aligned}
& \int_{S_{t}^{x}} \omega(\sigma)|\nabla f| \mathrm{d} \sigma \leq \eta t \int_{S_{t}^{x}} \omega(\sigma) \mathrm{d} \sigma \\
& \leq(2 \eta)\left(r_{x} \int_{S_{t}^{x}} \omega(\sigma) \mathrm{d} \sigma\right) \\
& \leq(2 \eta) \omega\left(B_{2 r_{x}}^{x}\right) \leq(6 C) \\
& \leq(2 \eta)^{\gamma-1} \omega\left(B_{t / 5}^{x}\right) \leq \eta C_{1} \omega\left(B_{t / 5}^{x}\right) .
\end{aligned}
$$

Again by means of Banach process and by virtue of (6) we get

$$
\sum_{v=1}^{N} \int_{S_{v}^{n}} \omega(\sigma)|\nabla f| \mathrm{d} \sigma \leq \eta \cdot C_{1} \omega(D)
$$

where $S_{v}^{n}$ is the surface of balls in the second covering. Combining the spherical surfaces $S_{v}^{\prime}$ and $S_{v}^{\prime \prime}$ we get that the open balls system cover the closed set $O_{f}$. Then a finite subcovering may be choosing from it. Let they be the balls $B_{1}, B_{2}, \cdots, B_{x}$ and their surfaces is $S_{1}, S_{2}, \cdots, S_{N}$.

We get from inequalities (3) and (5)

$$
\sum_{v=1}^{N} \int_{S_{v}}|\nabla f| \omega(\sigma) \mathrm{d} \sigma \leq\left[C_{1} \omega(\bar{D})+C_{0}\right] \eta .
$$

Put now $\varepsilon=\left[C_{1} \omega(\bar{D})+C_{0}\right] \eta$.

Following [2], assume

$$
\varepsilon=\frac{\omega(D)(\underset{G}{\operatorname{osc} u})}{R^{2}}
$$

and according to lemma 1 well find the balls $B_{1}, B_{2}, \cdots, B_{x}$ for given and exclude then from the domain $G$. Put $D^{*}=D / \bigcup_{v=1}^{N} B_{v}$ intersect with $G^{*}$ a closed spherical layer

$$
R\left(1+\frac{1}{4}\right) \leq|x| \leq R\left(1+\frac{1}{4}\right)
$$

We denote the intersection by $G^{\prime}$. We can assume that the function $u(x)$ is defined in some $\delta$ vicinity $G_{\delta}^{\prime}$ of set $G^{\prime}$. Take $\delta<\frac{R}{4}$ so that

$$
\underset{G_{\delta}^{\prime}}{\operatorname{osc}} u \leq \underset{G}{\operatorname{osc}} u \text {. }
$$

On a closed set $G^{\prime}$ we have $\nabla f \neq 0$. Consider on $G_{\delta}^{\prime}$ the equation system

$$
\frac{\mathrm{d} x}{\mathrm{~d} t}=u_{x}
$$

Let $S$ a such from surface that it touches to field direction at any his point, then

$$
\int_{S}\left|\frac{\partial u}{\partial n}\right| \mathrm{d} \sigma=0
$$

since $\frac{\partial u}{\partial n}$ is identically equal to zero at $S$.

We shall use it in constructing the needed surface of $\Sigma$. Tubular surfaces whose generators will be the trajectories of the system (10) constitute the basis of $\Sigma$.

They will add nothing to the integral we are interested in. These surfaces will have the form of thin tubes that cover $G^{\prime}$. Then we shall put partitions to some of these tubes. Lets construct tubes. Denote by $E$ the intersection of $G^{\prime}$ with sphere $|x|=R\left(1+\frac{3}{4}\right)$.

Let $N$ be a set of points $E$. Where field direction of system (10) touches the sphere $|x|=R\left(1+\frac{3}{4}\right)$. Cover $N$ with such an open on the sphere $|x|=R\left(1+\frac{3}{4}\right)$ set $F$ that

$$
\int_{F} \omega(x)\left|\frac{\partial u}{\partial n}\right| \mathrm{d} \sigma \leq \frac{\omega(G)\left(\begin{array}{c}
\text { osc } u \\
D
\end{array}\right)}{R^{2}} .
$$

It will be possible if on $N \frac{\partial u}{\partial R} \equiv 0$.

Put $E^{\prime}=E / F$. Cover $E^{\prime}$ on the sphere by a finite number of open domains with piece-wise smooth boundaries. We shall call them cells. We shall control their diameters in estimation of integrals that we need. The surface remarked by the trajectories lying in the ball $|x| \leq \frac{7}{4} R$ and passing through the bounds of cells we shall call tube.

So, we obtained a finite number of tubes. The tube is called open if not interesting this tube one can join by a broken line the point of its corresponding cell with a spherical layer $\frac{5}{4} R-\delta<|x|<\frac{7}{4} R$. Choose the diameters of cells so small that the trajectory beams passing through each cell, could differ no more than $\frac{\delta}{2 n}$.

By choose of cells diameters the tubes will be contained in

$$
\frac{5}{4} R-\delta<|x|<\frac{5}{4} R
$$

Let also the cell diameter be chosen so small that the surface that is orthogonal to one trajectory of the tube intersect the other trajectories of the tube at an angle more than $\frac{\pi}{4}$. 
Cut off the open tube by the hypersurface in the place where it has been imbedded into the layer

$$
\frac{5}{4} R-\frac{\delta}{2}<|x|<\frac{5}{4} R
$$

at first so that the edges of this tube be embedded into this layer.

Denote these cut off tubes by $T_{1}, T_{2}, \cdots, T_{S}$. If each open tube is divided with a partition, then a set-theoretical sum of closed tubes, tubes $T_{1}, T_{2}, \cdots, T_{S}$ their partitions spheres $S_{1}, S_{2}, \cdots, S_{N}$ and the set $F$ on the sphere $|x|=\frac{7}{4} R$ divides the spheres $|x|=R$ and $|x|=2 R$. Note that $\int_{S} \omega\left|\frac{\partial u}{\partial n}\right| \mathrm{d} \sigma$ along the surface of each tube equals to zero, since $\frac{\partial u}{\partial n}$ identically equals to zero.

Now we have to choose partitions so that the integral $\int_{S} \omega\left|\frac{\partial u}{\partial n}\right| \mathrm{d} \sigma \quad$ was of the desired value. Denote by $U_{i}$ the domain bounded by $T_{i}$ with corresponding cell and hypersurface cutting off this tube. We have $U_{i} \cap U_{j}=\varnothing$ and therefore

$$
\sum_{i=1}^{m} \omega\left(U_{i}\right)<2 \omega(D)
$$

Consider a tube $T_{i}$ and corresponding domain $U_{i}$. Choose any trajectory on this tube. Denote it by $L_{i}$. The length $\mu_{i} L_{i}$ of the curve $L_{i}$ satisfies the inequality

$$
\mu_{i} L_{i} \geq \frac{R}{2}
$$

On $L_{i}$ introduce a parameter in $l$-length of the are counted from cell. By $\sigma_{i}(l)$ denote the cross-section by $U_{i}$ hypersurface passing thought the point, corresponding to $l$ and orthogonal to the trajectory $L_{i}$ at this point. Let the diameter of cells be so small

$$
\int_{L_{i}} \mathrm{~d} l \int_{\sigma_{i}(l)} \omega(x) \mathrm{d} \sigma<2 \omega\left(U_{i}\right) .
$$

Then by Chebyshev inequality a set $H$ points $l \in L_{i}$ where

$$
\int_{\sigma_{i}(l)} \omega(x) \mathrm{d} \sigma>\frac{8}{R} \omega\left(U_{i}\right)
$$

satisfies the inequality $\mu_{i} H<\frac{R}{4}$ and hence by virtue of (13) for $E=L_{i} / H$ it is valid and

$$
\mu_{1} E>\frac{R}{4} .
$$

At the points of the curve $L_{i}$ the derivative $\frac{\partial u}{\partial l}$ preserves its sign, and therefore

$$
\int_{E}\left|\frac{\partial u}{\partial l}\right| \mathrm{d} l \leq \int_{L_{i}}\left|\frac{\partial u}{\partial l}\right| \mathrm{d} l \leq \underset{D_{\delta}^{\prime}}{\operatorname{osc} u}
$$

Hence, by using (15) and a mean value theorem for one variable function we find that there exists $l_{0} \in E$

$$
\left\|\frac{\partial u}{\partial l}\right\|_{l=l_{0}} \leq \frac{4}{R} \underset{D_{\delta}^{\prime}}{o s c u}
$$

But on the other hand

$$
\left\|\frac{\partial u}{\partial l}\right\|_{l=l_{0}}=|\nabla u|_{l=l_{0}} .
$$

Together with (16) it gives

$$
|\nabla u|_{l=l_{0}} \int_{\sigma_{i}\left(l_{0}\right)} \omega(x) \mathrm{d} \sigma \leq \frac{8}{R} \frac{4}{R} \omega\left(U_{i}\right)(\underset{D}{\operatorname{osc} u})
$$

Now, let the diameter of cells be still so small that

$$
\int_{\sigma_{i}\left(l_{0}\right)} \omega(x)|\nabla u| \mathrm{d} \sigma \leq \frac{16 \times 4}{R} \omega\left(U_{i}\right)(\underset{D}{\operatorname{osc} u})
$$

(we can do it, since the derivatives $\frac{\partial u}{\partial x_{i}}$ are uniformly continuous). Therefore according to (12)

$$
\sum_{i=1}^{S} \int_{\sigma_{i}\left(l_{0}\right)} \omega(x)|\nabla u| \mathrm{d} \sigma \leq \frac{16 \times 4}{R} \omega\left(U_{i}\right)(\underset{D}{\operatorname{osc} u})
$$

Now by $\Sigma$ we denote a set-theoretic sum of all open tubes all thought tubes $T_{i}$ all $\sigma_{i}\left(l_{0}\right)$ all spheres $S_{i}$ and sets $F$ on the sphere $|x|=\frac{7}{4} R$.

Then, we get by Equations (3), (9), (11) and (17)

$$
\int_{\Sigma} \omega(x)\left|\frac{\partial u}{\partial n}\right| \mathrm{d} \sigma \leq K \frac{\omega(D)(\underset{D}{\operatorname{osc} u})}{R^{p}} .
$$

The lemma is proved.

Denote by $W_{2, \omega}^{1}(D)$ the Banach space of the functions $u(x)$ defined in $D$ with the finite norm

$$
\|u\|_{W_{2, \omega}^{1}(D)}=\left(\int_{D} \omega\left(u^{2}+\sum_{i=1}^{n} u_{i}^{2}\right) \mathrm{d} x\right)^{\frac{1}{2}},
$$

and let $\stackrel{o}{W}_{2, \omega}^{1}(D)$ be a completion of $C_{0}^{\infty}(D)$ by the norm of the space $W_{2, \omega}^{1}(D)$.

By $m_{H}^{s}(A)$ we'll denote the Hausdorff measure of the set $A$ of order $s>0$. Further everywhere the notation $C(\cdots)$ means, that the positive constant $C$ depends only on the content of brackets. 


\section{Main Results}

Theorem 1. Let $D$ be a bounded domain in $\mathbb{E}_{n}$, $E \subset \bar{D}$ be a compact. If with respect to the coefficients of the operator $L$ the conditions (2)-(5) are fulfilled, then for removability of the compact $E$ with respect to the Equation (1) in the space $C_{\omega}^{\lambda}(D)$ it sufficies that

$$
m_{H}^{n-2+\lambda}(E)=0 .
$$

Proof. At first we show that without loss of generality we can suppose the condition $\partial D \in C^{1}$ is fulfilled. Suppose, that the condition (7) provides the removability of the compact $E$ for the domains, whose boundary is the surface of the class $C^{1}$, but $\partial D \in C^{1}$ and by fulfilling (7) the compact $E$ is not removable. Then the problem (6) has non-trivial solution $u(x)$, moreover $\left.u\right|_{E}=f(x)$ and $f(x) \neq 0$. We always can suppose the lowest coefficients of the operator $L$ are infinitely differentiable in $D$. Moreover, without loss of generality, we'll suppose that the coefficients of the operator $L$ are extended to a ball $B \supset \bar{D}$ with saving the conditions (2)(5). Let $f^{+}(x)=\max \{f(x), 0\}, f^{-}(x)=\min \{f(x), 0\}$, and $u^{ \pm}(x)$ be generalized by Wiener (see [8]) solutions of the boundary value problems

$$
L u^{ \pm}=0, x \in D / E ;\left.u^{ \pm}\right|_{\partial D / E}=0 ;\left.u^{ \pm}\right|_{E}=f^{ \pm} .
$$

Evidently, by $u(x)=u^{+}(x)+u^{-}(x)$. Further, let $D^{\prime}$ be such a domain, that $\partial D^{\prime} \in C^{1}, \bar{D} \subset D^{\prime}, \bar{D}^{\prime} \subset B$, and $\vartheta^{ \pm}(x)$ be solutions of the problems

$$
\begin{aligned}
& L \vartheta^{ \pm}=0, x \in D^{\prime} \backslash E ;\left.\vartheta^{ \pm}\right|_{\partial D^{\prime}}=0 ; \\
& \left.\vartheta^{ \pm}\right|_{E}=f^{ \pm} ; \vartheta^{ \pm}(x) \in C_{\omega}^{\lambda}\left(D^{\prime}\right) .
\end{aligned}
$$

By the maximum principle for $x \in D$

$$
0 \leq u^{+}(x) \leq \vartheta^{+}(x), \vartheta^{-}(x) \leq u^{-}(x) \leq 0 .
$$

But according to our supposition $\vartheta^{+}(x) \equiv \vartheta^{-}(x) \equiv 0$. Hence, it follows, that $u(x) \equiv 0$. So, we'll suppose that $\partial D \in C^{1}$. Now, let $u(x)$ be a solution of the problem (6), and the condition (7) be fulfilled. Give an arbitrary $\varepsilon>0$. Then there exists a sufficiently small positive number $\delta$ and a system of the balls $\left\{B_{r_{k}}\left(x^{k}\right)\right\}, k=1,2, \cdots$, such that $r_{k}<\delta, E \subset \bigcup_{k=1}^{\infty} B_{r_{k}}\left(x^{k}\right)$ and

$$
\sum_{k=1}^{\infty} r_{k}^{n-2+\lambda}<\varepsilon
$$

Consider a system of the spheres $\left\{B_{2 r_{k}}\left(x^{k}\right)\right\}$, and let $D_{k}=D \cap B_{2 r_{k}}\left(x^{k}\right), k=1,2, \cdots$, . Without loss of generality we can suppose that the cover $\left\{B_{2 r_{k}}\left(x^{k}\right)\right\}$ has a finite multiplicity $a_{0}(n)$. By lemma for every $k$ there exists a piece-wise smooth surface $\Sigma_{k}$ dividing in $D_{k}$ the spheres $S_{r_{k}}\left(x^{k}\right)$ and $S_{2 r_{k}}\left(x^{k}\right)$, such that

$$
\int_{\Sigma_{k}} \omega\left|\frac{\partial u}{\partial v}\right| \mathrm{d} s \leq \underset{D_{k}}{\operatorname{osc}} u \frac{\omega\left(D_{k}\right)}{r_{k}^{2}} .
$$

Since $u(x) \in C_{\omega}^{\lambda}(D)$, there exists a constant $H_{1}>0$ depending only on the function $u(x)$ such that

$$
\underset{D_{k}}{\operatorname{osc}} \omega u \leq H_{1}\left(2 r_{k}\right)^{\lambda} \text {. }
$$

Besides,

$$
\omega\left(D_{k}\right) \leq \operatorname{mes}_{n} B_{2 r_{k}}\left(x^{k}\right)=\Omega_{n} 2^{n} r_{k}^{n} ; k=1,2, \cdots,
$$

where $\Omega_{n}=\operatorname{mes}_{n} B_{1}(0)$. Using (10) and (11) in (9), we get

$$
\int_{\Sigma_{k}} \omega\left|\frac{\partial u}{\partial v}\right| \mathrm{d} s \leq C_{1} r_{k}^{n-2+\lambda} ; k=1,2, \cdots,
$$

where $C_{1}=K H_{1} 2^{n+\lambda}$.

Let $D_{\Sigma}$ be an open set situated in $D \backslash E$ whose boundary consists of unification of $\Sigma$ and $\Gamma$, where

$$
\Sigma=\bigcup_{k=1}^{\infty} \Sigma_{k}, \Gamma=\partial D \backslash \bigcup_{k=1}^{\infty} D_{k}^{+},
$$

$D_{k}^{+}$is a part of $D_{k}$ remaining after the removing of points situated between $\Sigma$ and $S_{2 r_{k}}\left(x^{k}\right) ; k=1,2, \cdots$. Denote by $D_{\Sigma}^{\prime}$ the arbitrary connected component $D_{\Sigma}$, and by $\mathcal{M}$ we denote the elliptic operator of divergent structure

$$
\mathcal{M}=\sum_{i, j=1}^{n} \frac{\partial}{\partial x_{i}}\left(a_{i j}(x) \frac{\partial}{\partial x_{j}}\right) .
$$

According to Green formula for any functions $z(x)$ and $W(x)$ belonging to the intersection $C^{2}\left(D_{\Sigma}^{\prime}\right) \cap C^{1}\left(\bar{D}_{\Sigma}^{\prime}\right)$, we have

$$
\int_{D_{\Sigma}^{\prime}}(z \mathcal{M} \beta-\beta \mathcal{M} z) \mathrm{d} x=\int_{\partial D_{\Sigma}^{\prime}}\left(z \frac{\partial \beta}{\partial v}-\beta \frac{\partial z}{\partial v}\right) \mathrm{d} s .
$$

Since $\partial D \in C^{1}$, then

$$
u(x) \in C^{1}\left(D_{\Sigma}\right) \cap C^{1}\left(\bar{D}_{\Sigma}^{\prime}\right)
$$

(see [9]). From (13) choosing the functions $z=1, \beta=\omega u^{2}$ we have

$$
\int_{D_{\Sigma}} \mathcal{M}\left(\omega u^{2}\right) \mathrm{d} x=2 \int_{\partial D_{\Sigma}^{\prime}} \omega u \frac{\partial u}{\partial v} \mathrm{~d} s+\int_{\partial D_{\Sigma}} \omega_{x_{i}} u^{2} \mathrm{~d} s .
$$

But $|u(x)| \leq M<\infty$ for $x \in \bar{D}$. Let's assume that the condition

$$
\omega_{x_{i}}<c \omega .
$$

is fulfilled. By virtue of condition $(*)$ and

$$
\int_{\partial D_{\Sigma}} \omega u^{2} \mathrm{~d} s<C_{3} M \varepsilon
$$

subject to (12) and (8) we conclude 


$$
\begin{aligned}
& \int_{D_{\Sigma}^{\prime}} \mathcal{M}\left(\omega u^{2}\right) \mathrm{d} x \\
& \leq 2 M a_{0} \sum_{k=1}^{\infty} \int_{\Sigma_{k}} \omega\left|\frac{\partial u}{\partial v}\right| \mathrm{d} s+\int_{D_{\Sigma}^{\prime}} \omega u^{2} \mathrm{~d} x \\
& \leq 2 M a_{0} C_{1} \sum_{k=1}^{\infty} r_{k}^{n-2+\alpha}+\varepsilon M C_{2}<C_{3} \varepsilon,
\end{aligned}
$$

where $C_{3}=2 M a_{0} C_{1}$.

On the other hand

$$
\begin{aligned}
& \mathcal{M}\left(\omega u^{2}\right)=6 u \omega \mathcal{M}(u) \\
& +2 \sum_{i, j=1}^{n} \omega a_{i j} u_{i} u_{j}+(2 u+1) \sum_{i, j=1}^{n} a_{i j} u_{x_{j}} \omega_{x_{i}} \\
& +\sum_{i, j=1}^{n} \frac{\partial a_{i j}}{\partial x_{i}} u \omega_{x_{j}}+\sum_{i, j=1}^{n} a_{i j} u \omega_{x_{i} x_{j}}
\end{aligned}
$$

and besides,

$$
\mathcal{M} u=\mathcal{L} u-\sum_{i=1}^{n} d_{i}(x) u_{i}+c(x) u-b(x, u, \nabla u),
$$

where $d$

$$
{ }_{i}(x)=\sum_{j=1}^{n} \frac{\partial a_{i j}(x)}{\partial x_{j}}-b_{i}(x), i=1, \cdots, n .
$$

It is evident that by virtue of conditions (3)-(4) $\left|d_{i}(x)\right| \leq d_{0}<\infty ; i=1, \cdots, n$. Thus, from (13) we obtain

$$
\begin{aligned}
& 6 \int_{D_{\Sigma}^{\prime}} u \omega \sum_{i=1}^{n} d_{i}(x) u_{i} \mathrm{~d} x-6 \int_{D_{\Sigma}^{\prime}} u^{2} c(x) \mathrm{d} x \\
& +2 \int_{D_{\Sigma}^{\prime}} \sum_{i, j=1}^{n} \omega(x) a_{i j} u_{i} u_{j} \mathrm{~d} x \\
& +(2 u+1) \int_{D_{\Sigma}^{\prime}} \sum_{i, j=1}^{n} a_{i j} u_{j} \omega_{x_{i}} \mathrm{~d} x \\
& +\int_{D_{\Sigma}^{\prime}} \sum_{i, j=1}^{n} \frac{\partial a_{i j}}{\partial x_{j}} u \omega_{x_{i}} \mathrm{~d} x+|\nabla u|^{2} \mathrm{~d} x \\
& +\int_{D_{\Sigma}^{\prime}} \sum_{i, j=1}^{n} a_{i j} u \omega_{x_{i} x_{j}} \mathrm{~d} x+b(x, u, \nabla u)<C_{3} \varepsilon .
\end{aligned}
$$

Let's estimate the nonlinear member on the right part applying the inequality

$$
\begin{aligned}
& \int_{D_{\Sigma}^{\prime}} b(x, u, \nabla u) \mathrm{d} x \\
& \leq \int_{D_{\Sigma}^{\prime}} g(x) \omega(x)|\nabla u| \mathrm{d} x \\
& \leq \frac{1}{2 \alpha} \int_{D_{\Sigma}^{\prime}} g^{2}(u) \mathrm{d} x+\int_{D_{\Sigma}^{\prime}} \omega^{2}(x)|\nabla u|^{2} \mathrm{~d} x
\end{aligned}
$$

Hence, for any $\alpha>0$ applying Cauchy inequality we have

$$
\begin{aligned}
& 2 \gamma \int_{D_{\Sigma}^{\prime}} \omega|\nabla u|^{2} \mathrm{~d} x \\
& <6 d_{0} \int_{D_{\Sigma}^{\prime}} \omega|u|\left|u_{i}\right| \mathrm{d} x+6 \int_{D_{\Sigma}^{\prime}} u^{2} \omega(x) \\
& +(2 u+1) \int_{D_{\Sigma}^{\prime}} a_{i j} u_{j} \omega_{x_{i}} \mathrm{~d} x \\
& +d_{0} \int_{D_{\Sigma}^{\prime}} u \omega_{x_{i}}^{2} \mathrm{~d} x+\int_{D_{\Sigma}^{\prime}} a_{i j} u \omega_{x_{i} x_{j}}+C_{3} \varepsilon \\
& \leq 6 \frac{d_{0}}{\varepsilon} \int_{D_{\Sigma}^{\prime}}|u|^{2} \mathrm{~d} x+6 \frac{d_{0} \varepsilon}{2} \int_{D_{\Sigma}^{\prime}} \omega^{2}|\nabla u|^{2} \mathrm{~d} x \\
& +(2 n+1) \int_{D_{\Sigma}^{\prime}} u_{j} \omega \mathrm{d} x+d_{0} \int_{D_{\Sigma}^{\prime}} u \omega \mathrm{d} x+\gamma C_{4} \varepsilon \\
& \leq 6 \frac{d_{0}}{\varepsilon} M m e s_{n} D+\frac{(2 M+1) \gamma}{\varepsilon} m e s_{n} D \\
& +d_{0} M \omega(D)+\gamma C_{4} M \omega(D)+C_{3} \varepsilon .
\end{aligned}
$$

If we'll take into account that

$$
\left|\omega_{x_{i} x_{j}}\right|<C_{4} \omega(x),
$$

then from here we have that

$$
\int_{D_{\Sigma}^{\prime}} \omega^{2}|\nabla u|^{2} \mathrm{~d} x \leq C_{5},
$$

where

$$
\begin{aligned}
C_{5} & =\left(6 d_{0}+(2 M+1)\right) \text { Mmes }_{n} D \\
& +\left(d_{0} M+\gamma C_{4} M\right) \omega(D)+\frac{C_{3}}{\gamma} .
\end{aligned}
$$

Without loss of generality we assume that $\varepsilon \leq 1$. Hence we have

$$
\int_{D} \omega^{2}|\nabla u|^{2} \mathrm{~d} x \leq C_{6} .
$$

Thus $u(x) \in W_{2, \omega}^{1}(D)$. From the boundary condition and $\operatorname{mes}_{n-1}(\partial D \cap E)=0$ we get $u(x) \in W_{2, \omega}^{1}(D)$. Now, let $\sigma \geq 2$ be a number which will be chosen later, $D_{\Sigma}^{+}=\left\{x: x \in D_{\Sigma}^{\prime}, u(x)>0\right\}$. Without loss of generality, we suppose that the set $D_{\Sigma}^{+}$isn't empty. Supposing in (13) $z=1, \beta=\omega u^{\sigma}$, we get

$$
\begin{aligned}
\int_{D_{\Sigma}^{+}} \mathcal{M}\left(\omega u^{\sigma}\right) \mathrm{d} x & =\sigma \int_{\partial D_{\Sigma}^{+}}\left(\omega_{v} u^{\sigma}+\sigma u^{\sigma-1} \frac{\partial u}{\partial v}\right) \mathrm{d} s \\
& \leq M^{\sigma} \int_{\partial D_{\Sigma}^{+}} \omega \mathrm{d} s+\sigma M^{\sigma-1} \int_{\partial D_{\Sigma}^{ \pm}}\left|\frac{\partial u}{\partial v}\right| \mathrm{d} s \\
& \leq C_{5}\left(a_{0}, M, \sigma, C_{1}\right) \varepsilon .
\end{aligned}
$$

But, on the other hand 


$$
\begin{aligned}
& \mathcal{M}\left(u^{\sigma}\right)=\sum_{i, j=1}^{n} \frac{\partial}{\partial x_{i}}\left(a_{i j} \frac{\partial \omega u^{\sigma}}{\partial x_{j}}\right)+b(x, u, \nabla u) \\
& =\sum_{i, j=1}^{n} \frac{\partial}{\partial x_{i}}\left(a_{i j} \omega\left(\sigma u^{\sigma-1} \frac{\partial u}{\partial x_{j}}\right)+\sum_{i, j=1}^{n} \frac{\partial}{\partial x_{i}}\left(a_{i j} \omega_{x_{i}} \frac{\partial u^{\sigma}}{\partial x_{j}}\right)\right) \\
& +b(x, u, \nabla u) \\
& =\sum_{i, j=1}^{n} \frac{\partial}{\partial x_{i}}\left(a_{i j} \omega \sigma u^{\sigma-1} \frac{\partial u}{\partial x_{j}}\right)+\sum_{i, j=1}^{n} \frac{\partial}{\partial x_{i}}\left(a_{i j} \sigma u^{\sigma-1} \omega x \frac{\partial u}{\partial x_{j}}\right) \\
& +b(x, u, \nabla u) \\
& =\sigma \omega u^{\sigma-1} \mathcal{M}(u)+\sigma \omega \frac{\partial}{\partial x_{i}}\left(a_{i j} u^{\sigma-1} \frac{\partial u}{\partial x_{j}}\right) \\
& +\sigma u^{\sigma-1} \frac{\partial}{\partial x_{i}}\left(a_{i j} \omega \frac{\partial u}{\partial x_{j}}\right)+b(x, u, \nabla u)+\beta \\
& =\sigma \omega u^{\sigma-1} \mathcal{M}(u)+\sigma \omega u^{\sigma-1} \frac{\partial}{\partial x_{i}}\left(a_{i j} \frac{\partial u}{\partial x_{j}}\right) \\
& +\sigma \omega a_{i j} u_{x_{j}}(\sigma-1) u^{\sigma-2} u_{x_{i}}+\sigma u^{\sigma-1} \omega_{x_{i}}\left(a_{i j} \frac{\partial u}{\partial x_{j}}\right) \\
& +\sigma u^{\sigma-1} \omega \frac{\partial}{\partial x_{i}}\left(a_{i j} \frac{\partial u}{\partial x_{j}}\right)+\beta+b(x, u, \nabla u) \\
& =3 \sigma \omega u^{\sigma-1} M(u)+\sigma(\sigma-1) a_{i j} u x_{i} u_{x_{j}} u^{\sigma-2} \omega \\
& +\sigma u^{\sigma-1} \omega_{x_{i}} a_{i j} u_{x_{j}}+\beta+b(x, u, \nabla u) \\
& =\sigma \int_{D_{\Sigma}^{+}} d_{i}(x) u_{x_{i}} u \omega \mathrm{d} x-\sigma(\sigma-1) \int_{D_{\Sigma}^{+}} u^{\sigma} \omega(x) c(x) \mathrm{d} x \\
& +\sigma(\sigma-1) \int_{D_{\Sigma}^{+}} \sum_{i, j=1}^{n} u^{\sigma-2} \omega(x) a_{i j} u_{i} u_{j} \mathrm{~d} x \\
& +(2 u+1) \int_{D_{\Sigma}^{+}} \sum_{i, j=1}^{n} a_{i j} u_{j} \omega_{x_{j}} u^{\sigma-1}+b(x, u, \nabla u) .
\end{aligned}
$$

Hence, we conclude

$$
\begin{aligned}
& \sigma(\sigma-1) \int_{D_{\Sigma}^{+}} \omega^{2} u^{\sigma-2}|\nabla u|^{2} \mathrm{~d} x \\
& \leq d_{0} \int_{D_{\Sigma}^{+}} u^{\sigma-1} \omega u_{i} \mathrm{~d} x+b(x, u, \nabla u) \\
& \leq d_{0} \int_{D_{\Sigma}^{+}} u^{\sigma-1} \omega u_{i} \mathrm{~d} x+b(x, u, \nabla u) \\
& \leq \frac{d_{0} \varepsilon}{2} \int_{D_{\Sigma}^{+}} u^{\sigma} \mathrm{d} x+b(x, u, \nabla u) .
\end{aligned}
$$

Let $D^{+}=\{x: x \in D, u(x)>0\}, D_{1}^{+}$be an arbitrary connected component of $D^{+}$. Subject to the arbitrariness of $\varepsilon$ from (16) we get

$$
(\sigma-1) \gamma \int_{D_{\Sigma}^{+}} \omega u^{\sigma-2}|\nabla u|^{2} \mathrm{~d} x \leq d_{0} \int_{D_{1}^{+}} \omega u^{\sigma-1} \sum_{i=1}^{n}\left|u_{i}\right| \mathrm{d} x .
$$

Thus, for any $\mu>0$

$$
\begin{aligned}
& (\sigma-1) \gamma \int_{D_{1}^{+}} \omega u^{\sigma-2}|\nabla u|^{2} \mathrm{~d} x \\
& \leq \frac{d_{0} \mu}{2} \int_{D_{1}^{+}} \omega u^{\sigma-2}\left(\sum_{i=1}^{n}\left|u_{i}\right|\right)^{2} \mathrm{~d} x+\frac{d_{0}}{2 \mu} \int_{D_{1}^{+}} \omega u^{\sigma} \mathrm{d} x \\
& \leq \frac{d_{0} \mu n}{2} \int_{D_{1}^{+}} \omega u^{\sigma-2}|\nabla u|^{2} \mathrm{~d} x+\frac{d_{0}}{2 \mu} \int_{D_{1}^{+}} \omega u^{\sigma} \mathrm{d} x .
\end{aligned}
$$

But, on the other hand

$$
\begin{aligned}
I & =-\sigma \sum_{i=1}^{n} \int_{D_{1}^{+}} x_{i} \omega u^{\sigma-1} u_{i} \mathrm{~d} x \\
& =-\sum_{i=1}^{n} \int_{D_{1}^{+}} x_{i} \omega\left(u^{\sigma}\right)_{i} \mathrm{~d} x=n \int_{D_{1}^{+}} \omega u^{\sigma} \mathrm{d} x
\end{aligned}
$$

and besides, for any $\beta>0$

$$
I=\frac{\sigma \beta}{2} \int_{D_{1}^{+}} r^{2} \omega u^{\sigma} \mathrm{d} x+\frac{\sigma}{2 \beta} \int_{D_{1}^{+}} u^{\sigma-2} \omega^{2}|\nabla u|^{2} \mathrm{~d} x
$$

Then

$$
I \leq \frac{\sigma \beta}{2} \int_{D_{1}^{+}} r^{2} \omega u^{\sigma} \mathrm{d} x+\frac{\sigma}{2 \beta} \int_{D_{1}^{+}} \omega^{2}|\nabla u|^{2} u^{\sigma-2} \mathrm{~d} x,
$$

where $r=|x|$. Denote by $k(D)$ the quantity $\sup _{x \in D}|x|$.

Without loss of generality we'll suppose, that $k(D)=1$. Then

$$
I \leq \frac{\sigma}{2 \beta} \int_{D_{1}^{+}} \omega u^{\sigma} \mathrm{d} x+\frac{\sigma}{2 \beta} \int_{D_{1}^{+}} \omega^{2} u^{\sigma-2}|\nabla u|^{2} \mathrm{~d} x .
$$

Thus,

$$
\left(n-\frac{\sigma \beta}{2}\right) \int_{D_{1}^{+}} \omega u^{\sigma} \mathrm{d} x+\frac{\sigma}{2 \beta} \int_{D_{1}^{+}} \omega^{2} u^{\sigma-2}|\nabla u|^{2} \mathrm{~d} x .
$$

Now, choosing $\beta=\frac{n}{\sigma}$, we finnaly obtain

$$
\int_{D_{1}^{+}} \omega u^{\sigma} \mathrm{d} x \leq \frac{\sigma^{2}}{n^{2}} \int_{D_{1}^{+}} \omega^{2} u^{\sigma-2}|\nabla u|^{2} \mathrm{~d} x .
$$

Subject to Equation (18) in Equation (17) ,we conclude

$$
\begin{aligned}
& (\sigma-1) \gamma \int_{D_{1}^{+}} \omega^{2} u^{\sigma-2}|\nabla u|^{2} \mathrm{~d} x \\
& \leq\left(\frac{d_{0} \varepsilon n}{2}+\frac{d_{0} \sigma^{2}}{2 \varepsilon n^{2}}\right) \int_{D_{1}^{+}} \omega^{2} u^{\sigma-2}|\nabla u|^{2} \mathrm{~d} x .
\end{aligned}
$$




$$
(\sigma-1) \gamma>\frac{d_{0} \mu n}{2}+\frac{d_{0} \sigma^{2}}{2 \mu n^{2}} .
$$

Then from Equations (18)-(20) it will follow that $u(x) \equiv 0$ in $D_{1}^{+}$, and thus $u(x) \equiv 0$ in $D$. Suppose that

$$
\mu=\frac{(\sigma-1) \gamma}{d_{0} n} .
$$

Then Equation (20) is equivalent to the condition

$$
n>\left(\frac{\sigma}{\sigma-1}\right)^{2}\left(\frac{d_{0}}{\gamma}\right)^{2} \text {. }
$$

At first, suppose that

$$
n>\left(\frac{d_{0}}{\gamma}\right)^{2} \text {. }
$$

Let's choose and fix such a big $\sigma \geq 2$ that by fulfilling (22) the inequality (21) was true. Thus, the theorem is proved, if with respect to $n$ the condition (22) is fulfilled. Show that it is true for any $n \geq 3$. For that, at first, note that if $k(D) \neq 1$, then condition (22) will take the form

$$
n>\left(\frac{d_{0} k(D)}{\gamma}\right)^{2} .
$$

Now, let the condition (22) be not fulfilled. Denote by $k$ the least natural number for which

$$
n+k>\left(\frac{d_{0}}{\gamma}\right)^{2} .
$$

Consider $(n+k)$-dimensional semi-cylinder

$$
D^{\prime}=D \times\left(-\delta_{0}, \delta_{0}\right) \times, \cdots, \times\left(-\delta_{0}, \delta\right),
$$

where the number $\delta_{0}>0$ will be chosen later. Since $\omega(D)=1$, then $\omega\left(D^{\prime}\right) \leq 1+\delta_{0} \sqrt{k}$. Let's choose and fix $\delta_{0}$ so small that along with the condition (23) the condition

$$
n+k>\left(\frac{d_{0} \omega\left(D^{\prime}\right)}{\gamma}\right)^{2} .
$$

was fulfilled too.

Let

$$
\begin{aligned}
& y=\left(x_{1}, \cdots, x_{n}, x_{n+1}, \cdots, x_{n+k}\right), \\
& E^{\prime}=\underbrace{E \times\left[-\delta_{0}, \delta_{0}\right] \times \cdots \times\left[-\delta_{0}, \delta_{0}\right]}_{k \text { times }}
\end{aligned}
$$

Consider on the domain $D^{\prime}$ the equation

$$
\begin{aligned}
\mathcal{L}_{\vartheta}^{\prime} & =\sum_{i, j=1}^{n} a_{i j}(x) \vartheta_{i j}+\sum_{i=1}^{k} \frac{\partial^{2} \vartheta}{\partial x_{n+i}^{2}} \\
& +\sum_{i=1}^{n} b_{i}(x) \vartheta_{i}+c(x) \vartheta=0 .
\end{aligned}
$$

It is easy to see that the function $\vartheta(y)=u(x)$ is a solution of the Equation (25) in $D^{\prime} / E^{\prime}$. Besides,

$$
m_{H}^{n+k-2+\lambda}\left(E^{\prime}\right)=\left(2 \delta_{0}\right)^{k} m_{H}^{n-2+\lambda}(E)=0,
$$

the function $\vartheta(y)$ vanishes on

$$
(\partial D \times \underbrace{\left[-\delta_{0}, \delta_{0}\right] \times \cdots \times\left[-\delta_{0}, \delta_{0}\right]}_{k \text { times }}) / E^{\prime} \text { and } \frac{\partial \vartheta}{\partial v^{\prime}}=0
$$

at $x_{n+i}= \pm \delta_{0}, i=1, \cdots, k$, where $\frac{\partial}{\partial v^{\prime}}$ is a derivative by the conormal generated by the operator $\mathcal{L}^{\prime}$. Noting that $\gamma\left(\mathcal{L}^{\prime}\right)=\gamma(\mathcal{L}), d_{0}\left(\mathcal{L}^{\prime}\right)=d_{0}(\mathcal{L})$ and subject to the condition (24), from the proved above we conclude that $\vartheta(y) \equiv 0$, i.e. $D^{\prime}$. The theorem is proved.

Remark. As is seen from the proof, the assertion of the theorem remains valid if instead of the condition (3) it is required that the coefficients $a_{i j}(x)(i, j=1, \cdots, n)$ have to satisfy in domain $D$ the uniform Lipschitz condition with weight.

Thus in this paper the sufficient condition for removability of the compact respect Newman problem for quasilinear equation in classes in the weight space of Holder functions is obtained.

\section{REFERENCES}

[1] L. Carleson, "Selected Problems on Exceptional Sets," D. Van Nostrand Company, Toronto, 1967, 126 p.

[2] E. I. Moiseev, "On Neumann Problems in Piecewise Smooth Domains," Differentsial'nye Uravneniya, Vol. 7, No. 9, 1971, pp. 1655-1656.

[3] E. I. Moiseev, "On Existence and Non-Existence Boundary Sets of Neumann Problem," Differentsial'nye Uravneniya, Vol. 9, No. 5, 1973, pp. 901-911.

[4] E. M. Landis, "To Question on Uniqueness of Solution of the First Boundary Value Problem for Elliptic and Parabolic Equations of the Second Order," Uspekhi Matematicheskikh Nauk, Vol. 33, No. 3, 1978, p. 151.

[5] V. A. Kondratyev and E. M. Landis, "Qualitative Theory of Linear Partial Differential Equations of Second Order," Modern Problems of Mathematics, Fundamental Directions, Partial Differential Equations, Vol. 3, Itogi Nauki i Tekhniki, Seriya, VINITI, 1988, pp. 99-212.

[6] I. T. Mamedov, "On Exceptional Sets of Solutions of Dirichlet Problem for Elliptic Equations of Second Order with Discontinuous Coefficients," Proceedings of Institute of Mathematics and Mechanics of Academy of Science of Azerbaijan, Vol. 3, No. 16, 1998, pp. 137-149.

[7] M. L. Gerver and E. M. Landis, "One Generalization of a Theorem on Mean Value for Multivariable Functions," Doklady Akademii Nauk USSR, Vol. 146, No. 4, 1962, pp. 761-764.

[8] E. M. Landis, "Second Order Equations of Elliptic and Parabolic Types," Nauka, 1971, 288 p. 
[9] D. Gilbarg and N. S. Trudinger, "Elliptic Partial Differential Equations of Second Order," Springer-Verlag, Berlin, 1977, 401 p. doi:10.1007/978-3-642-96379-7

[10] V. Kayzer and B. Muller, "Removable Sets for Heat Conduction," Vestnik, Moscow University, Moscow, 1973, pp. 26-32.

[11] V. A. Mamedova, "On Removable Sets of Solutions of Boundary Value Problems for Elliptic Equations of Second Order," Transactions of the NAS of Azerbaijan, Vol. 25, No. 1, 2005, pp. 101-106.

[12] T. S. Gadjiev and V. A. Mamedova, "On Removable Sets of Solutions of Second Order Elliptic and Parabolic Equations in Nondivergent Form," Ukrainian Mathematical Journal, Vol. 61, No. 11, 2009, pp. 1485-1496.

[13] T. Kilpelainen and X. Zhong, "Removable Sets for Continuous Solutions of Quasilinear Elliptic Equations," Proceedings of the American Mathmatical Society, Vol. 130, No. 6, 2002, pp. 1681-1688. doi:10.1090/S0002-9939-01-06237-2
[14] J. Diederich, "Removable Sets for Pointwise Solutions of Elliptic Partial Differential Equations," Transactions of the American Mathmatical Society, Vol. 165, 1972, pp. 333-352. doi:10.1090/S0002-9947-1972-0293235-X

[15] R. Harvey and J. Polking, "Removable Singularities of Solutions of Linear Partial Differential Equations," Acta Mathematica, Vol. 125, No. 1, 1970, pp. 39-56. doi:10.1007/BF02838327

[16] B. E. J. Dahlberg, "On Exceptional Sets at the Boundary for Subharmonic Functions," Arkiv för Matematik, Vol. 15, No. 2, 1977, pp. 305-312.

[17] A. V. Pokrovskii, "Removable Singularities of Solutions of Linear Uniformly Elliptic Second Order Equations," Funktsionalnyj Analiz i Prilozhenija, Vol. 42, No. 2, 2008, pp. 44-55.

[18] A. V. Pokrovskii, "Removable Singularities for Solutions of Second-Order Linear Uniformly Elliptic Equations in Non-Divergence Form," Mathematics Sbornik, Vol. 199, No. 6, 2008, pp. 137-160. 